\title{
The Fast and Continuous Railway Clearance Inspection Algorithm Based on Vehicle LiDAR Point Cloud
}

\author{
Dan ZHONG, Tonggang ZHANG ${ }^{1}$, Yuhui KAN, and Fugui XIE \\ Faculty of Geosciences and Environmental Engineering, Southwest Jiaotong \\ University, 611756 Chengdu, China
}

\begin{abstract}
Railway clearance inspection is the foundation work to ensure train safety operation. Aiming at the problem of time-consuming and low accuracy of clearance inspection based on vehicle LiDAR point cloud, this paper proposes a fast and continuous railway clearance inspection algorithm. Firstly, the preliminary clearance inspection is completed by constructing the axial alignment bounding box of the clearance polyhedron. Then fine clearance inspection based on the topology relationship between point cloud and polyhedron to provide an accurate judgment. Finally, the clearance distribution information is obtained by Euclidean clustering. Based on three representative datasets, the proposed algorithm is validated, and the experimental results show that the accuracy and efficiency are enhanced by $25.0 \%$ and $83.4 \%$ responsibly.
\end{abstract}

Keywords. Clearance polyhedron, vehicle LiDAR point cloud, railway, clearance inspection

\section{Introduction}

The railway clearance is the closed polyline that cannot be crossed by buildings, equipment and trains to ensure the safe operation. In the early days, contact method[1] was commonly applied. This method cannot be applied to railway electrification sections. In order to solve the problems of clearance inspection in the electrified sections, non-contact inspection method came into being. The visual image measurement is applied to clearance inspection [2-5]. With the rapid development of photoelectric technology, 3D laser mobile measurement equipment integrated with multiple sensors can quickly acquire high density and high precision vehicle LiDAR point cloud. The clearance inspection based on vehicle LiDAR point cloud has become a development direction of clearance inspection. At present, the clearance inspection based on vehicle LiDAR point cloud adopts section method, and widely applied in the field of highway[6]. Puente et al. [7] describes evaluating the vertical clearance of the highway tunnel. Gargoum et al. [8] carried out vertical clearance analysis for the whole section of highway.

In the field of railway, there are relatively few researches on the clearance inspection based on vehicle LiDAR point cloud. Zhou et al.[9] inspect tunnel clearance by the cross

${ }^{1}$ Tonggang ZHANG, Faculty of Geosciences and Environmental Engineering, Southwest Jiaotong University, 611756 Chengdu, China. Emails: tgzhang@swjtu.edu.cn 
section from the derived TIN from point cloud. It can check the clearance where the cross section locating, and other position is neglected. Niina et al. [10] proposed to project the original point cloud segments into cross sections continuously, and then visually judge the relationship between the cross section and the standard clearance . This method can obtain the cross section of point cloud, but it is inefficient. Based on the algorithms of Zhou et al.[9] and Niina et al. [10], this paper proposed a fast and continuous railway clearance inspection algorithm. The algorithm involves judging the relationship between the point cloud and the clearance polyhedron along the centerline, which can effectively complete the clearance inspection for all positions in the railway.

\section{Railway Continuous Clearance Inspection Based on Vehicle LiDAR Point Cloud}

Assuming the centerline is known, and the vehicle LiDAR point cloud has been converted to the railway coordinate system. Getting a corresponding clearance polyhedron and finishing the clearance inspection start from the starting point $\left(M_{0}\right)$ of the centerline until the end point $\left(M_{1}\right)$ to complete clearance inspection at arbitrary point $(M)$ of the centerline. When there is superelevation in railway curve segment, the clearance polyhedron rotates internally with the top of the outer rail as the axis instead of tilting the train toward the inside of the curve, internal rotation angle $\theta(\theta=\arctan (h /$ $\arctan (h / d)) . h$ is the superelevation, $d$ is the railway gauge. When the clearance width of the inside and outside of the curve section is considered ,the clearance polyhedron rotates inward at an angle of $\theta$ with the top of the inner rail as the axis and outward by an angle with the top of the outer rail as the axis, outward rotation angle $\beta\left(\beta=\arccos \frac{4000}{(5650+y) R}\right)$. R is the curve radius, $y$ is the height of catenary structure. The clearance inspection process of single-track railway is summarized in the Figure 1, that double-track railway is inspected in accordance with the single-track railway.

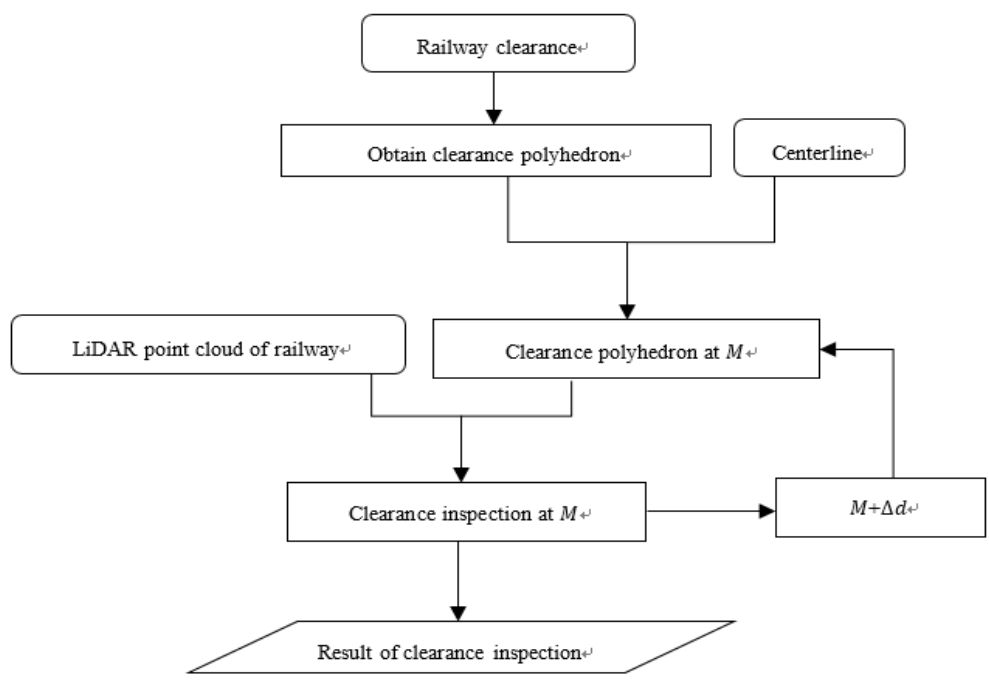

Figure 1. Railway continuous clearance inspection

Detailed steps of railway continuous clearance inspection based on vehicle LiDAR 
point cloud are as follows:

1) Stretch a certain length $(\Delta d)$ along the direction of mileage according to the clearance polygon to establish the railway clearance polyhedron.

2) Complete the clearance inspection of $M_{0} \sim M_{0}+\Delta d$ segment at the starting point $\left(M_{0}\right)$ of the centerline to obtain the invasive point cloud.

3) Let $M=M+\Delta d$ and repeat step 2) until the entire railway is inspected.

4) Obtain the distribution information of the invasive point cloud by Euclidean clustering and output the clearance inspection results.

\subsection{Railway Clearance Polyhedron}

Take the midpoint of the line between the top of the left rail and the right rail as the origin of the coordinate system $(O)$, the upward direction is the positive direction of the $Z$-axis, and the orthogonal with the $Z$-axis is the $Q$-axis. The 2D left-hand railway clearance coordinate system (2D RCC) is Established and shown in Figure 2.The 3D railway clearance coordinate (3D RCC) system is defined with its origin $(O)$ at the center point of the track, the $L$-axis along the rail route, $Z$-axis along upward direction, and $Q$-axis the orthogonal with the $Z$-axis and $L$-axis (Figure 3 ).

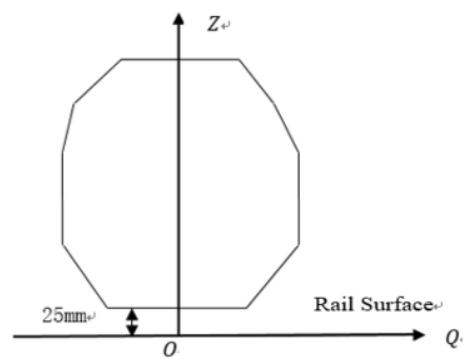

Figure 2. 2D Railway clearance coordinate system

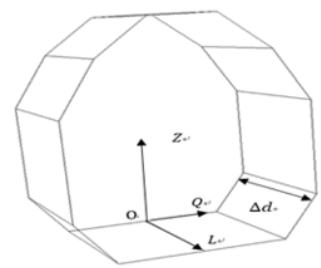

Figure 3. 3D railway clearance coordinate system

Railway clearance polyhedron is obtained by extending the clearance polygon a certain length $(\Delta d)$ along the $L$-axis in $3 \mathrm{D}$ RCC system.

\subsection{Clearance Inspection at Arbitrary Position}

As shown in Figure 4, the railway Clearance polyhedron is used to clearance inspect at $M$,which is the arbitrary position of the railway. 


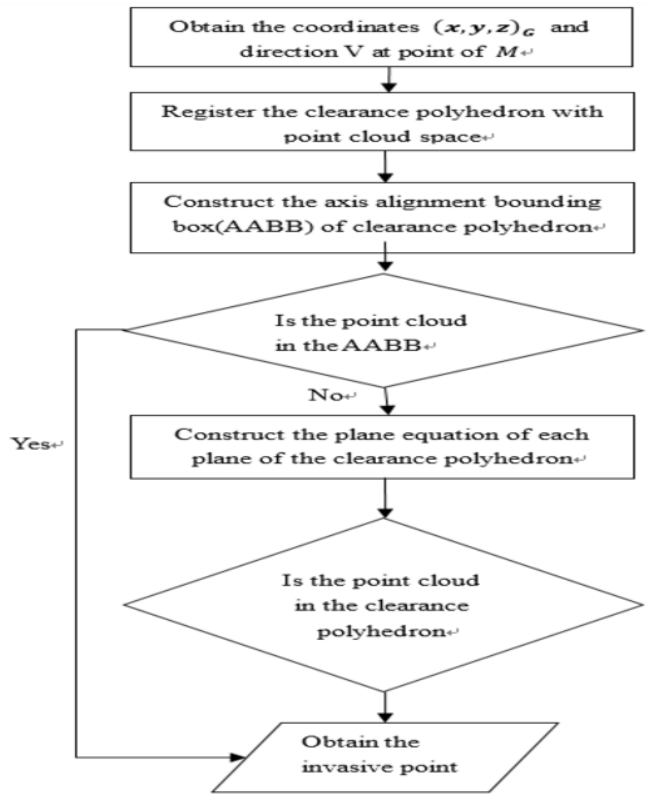

Figure 4. Clearance inspect at $M$

The detailed steps are given as follows:

1) Calculate the coordinates $(x, y, z)_{G}$ and 3D direction vector $(V)$ at point of $G$ in the railway coordinate system.

2) Find the precise position and pose of clearance polyhedron in point cloud by transform the clearance polyhedron. Let the point $O$ of the clearance polyhedron be at $G$, $L$-axis axis to $V$ direction, $Q$-axis parallel to the rail surface (Figure 5).

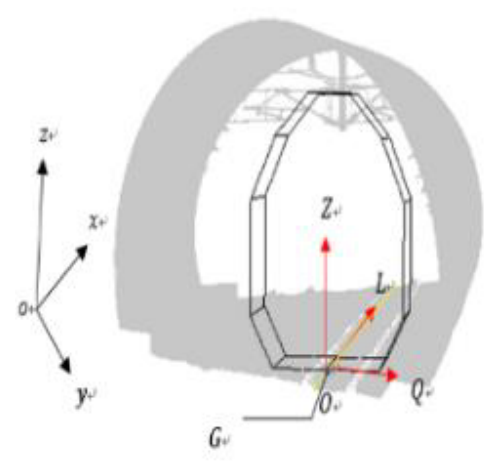

Figure 5. Register the clearance polyhedron with point cloud

3) Preliminary clearance inspection. The points located in Axially Aligned Bounding Boxes (AABB) of railway clearance polyhedron are evaluated as preliminary invasive point cloud.

4) Fine clearance inspection. According to the topological relationship between the points inside $\mathrm{AABB}$ and railway Clearance polyhedron to judge whether the point cloud invaded the clearance. If the point cloud is invaded, output the invasive point cloud. 


\subsubsection{Preliminary Clearance Inspection}

The point cloud is divided into the inner and outer parts of the axial alignment bounding box. The outer is non-invasion point cloud and the inner is the preliminary invasive point cloud. It can be seen from Figure 6 that the preliminary clearance inspection results cannot accurately express the clearance inspection results. The preliminary invasive point cloud requires detailed clearance inspecting.

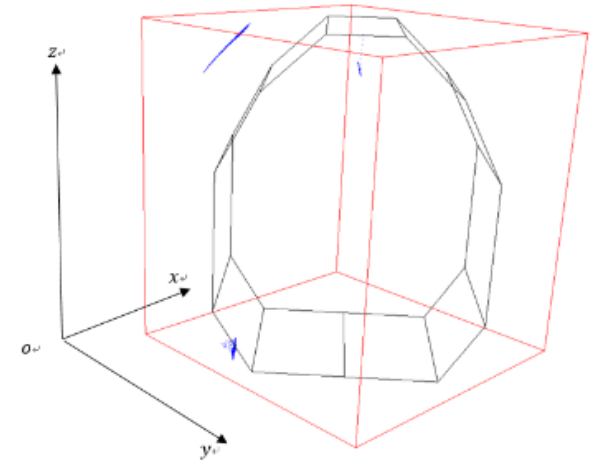

Figure 6. The Axially Aligned Bounding Boxes. The $o-x y z$ is the coordinate system of the point cloud, the cube is $\mathrm{AABB}$ (red), and the point cloud is the AABB (blue)

\subsubsection{Fine Clearance Inspection}

According to the real application, the shape of the railway clearance polyhedron may be different, but its surface consists of several planes.

For point $\mathrm{P}$ in the $\mathrm{AABB}, M_{P}$ is the mileage at point $\mathrm{P}$. If $M_{P} \in\left[M_{0}, M_{0}+\Delta d\right]$, the relationship between point $\mathrm{P}$ and each side is used to judge whether it is inside the clearance polyhedron. The specific judgment process is as follows:

1) Calculate the directed distance $d_{j}^{0}$ from $N$, gravity center of the railway clearance polyhedron, to $j$-th side, and the directed distance $d_{j}$ from $\mathrm{P}$ to $j$-th side.

2) If any $d_{j}$ and $d_{j}^{0}$ have different signs, $\mathrm{P}$ is outside the clearance polyhedron; if all $d_{j}$ and $d_{j}^{0}$ have same sign, $\mathrm{P}$ is inside the clearance polyhedron (Figure 7), and is marked as invasive point.

3) Repeat Step 1) and 2), until all points in AABB is judged.

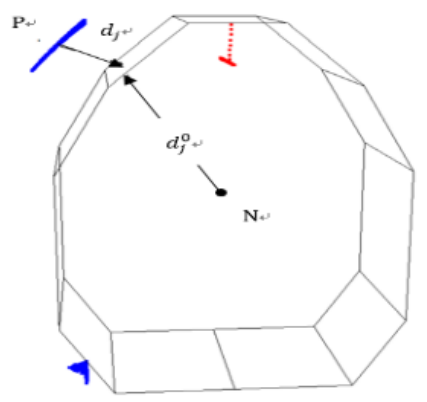

(a) The uninvaded point cloud inspection (blue)

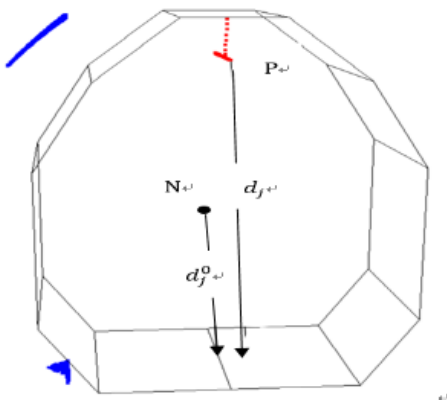

(b) The invaded point cloud inspection (red)

Figure 7.3D Clearance inspection diagram 
If the railway clearance polygon is not a convex polygon, it should be split into two convex polygons firstly, and then construct two adjacent clearance polyhedrons. Each point will be judge twice in two clearance polyhedrons. The specific process is as follows:

1) Make a counterclockwise sequence of polygon vertices, and judge whether the $k$ 'th point is concave in turn according to counterclockwise. The vertices of a polygon are represented by T, the value $E$ is the cross product at point $T_{k}$, the value $E$ is as follows:

$$
E=\overline{T_{k-1} T_{k}} \times \overline{T_{k-1} T_{k+1}}
$$

The vector of $\overline{T_{k-1} T_{k}}$ is the vector between the point before $k$ and $k$, the vector of $\overline{T_{k-1} T_{k+1}}$ is the vector between the point before $k$ and the point after the $k$. If the value $E$ is negative, $T_{k}$ is a concave point.Judge in turn until a second concave point is found to stop.

2) The judged convex points between the two concave points form a sub convex polygon in order.

3) The two concave points and the remaining undetermined points of the polygon form another sub convex polygon.

According to the method in Section 2.1, the two sub-convex polygons generated in steps 2) and 3) are generated into two clearance sub-polyhedron. The relationship between point $P$ and the two sub-polyhedron is judged according to the section 2.2.1. If $\mathrm{P}$ is judged in any one of them, it is marked invasive point.

\section{Experiment}

In order to test the performance of the proposed algorithm for railway clearance inspection, a simulation test of clearance inspection is designed. The results are expressed as the distribution of the invasive point cloud after the Euclidean clustering and time .of the clearance inspection. Before the test, the distribution of the invasive point cloud was manually set. The railway clearance inspection is carried out by using the proposed algorithm and the section clearance method. The accuracy of the clearance inspection algorithm is judged by whether the distribution of the invasive point cloud is found. The efficiency of the clearance inspection algorithm is judged by the clearance inspection time.

In this experiment, set parameters of polyhedron length $\Delta d=1 \mathrm{~m}$, the section is obtained at an interval of $1 \mathrm{~m}$ and the point cloud within the range of $0.01 \mathrm{~m}$ before and after the section is inspected by the method in Ref [9]. The visual section clearance method in Ref [10] takes the point cloud projection section by the segmented point cloud of $1 \mathrm{~m}$ successively. The Ref [9] directly calculated the distance between the section and the standard clearance after obtaining the section and Ref [10] judged the result by visual inspection after obtaining the section, so the time of the section clearance method in this experiment only record the time of obtaining the section from the point cloud. The algorithm of this paper record the completion time. In the experiment, the distance threshold is set to $1 \mathrm{~m}$ for Euclidean clustering of point cloud and the result of the clearance inspection is represented by the clustering result. 


\subsection{Experimental Data}

Three $500 \mathrm{~m}$ long railway point clouds, tunnel, platform and subgrade (Figure8), obtained by vehicle LiDAR system are adopted to test the three algorithms. The superelevation is $50 \mathrm{~mm}$ in curve section. The clearance polygon is shown in Figure 9.

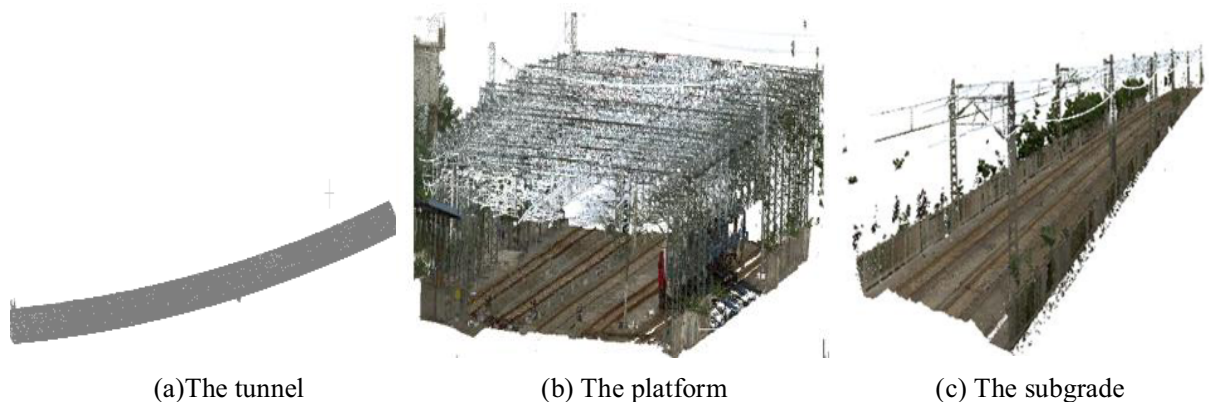

Figure 8. Railway point clouds

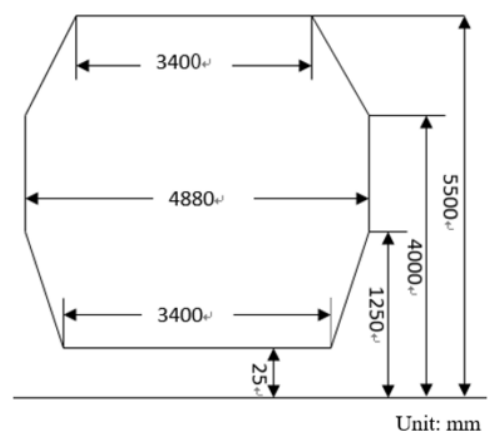

(a)The tunnel and subgrade

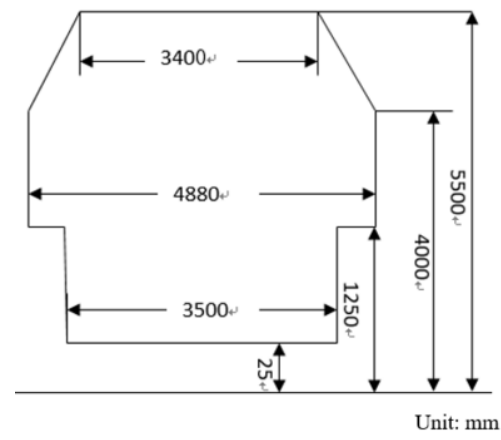

(b) The platform

Figure 9. The simulated standard clearance

The ground truth of invasive points is set manually. There are 10 invasive distribution in the tunnel, 12 invasive distribution in the platform, and 6 invasive distribution in the subgrade. Then the accuracy can be judged by comparing the known and found invasive points. The efficiency is measured by the consuming time.

\subsection{Experimental Result and Analysis}

The clearance inspection result of two algorithms are given in Figure 10. The number of invasive points given by them are shown in Table 1 . 


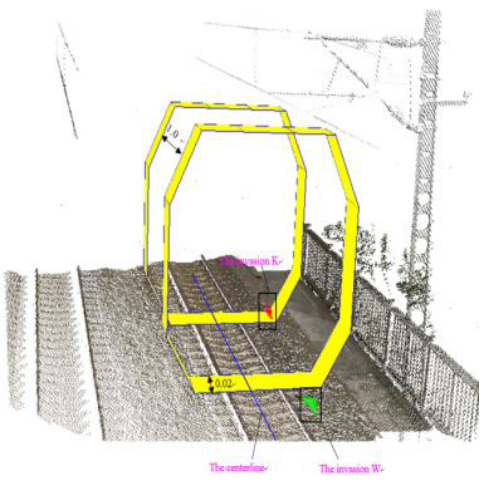

(a) The algorithm in Ref [9]

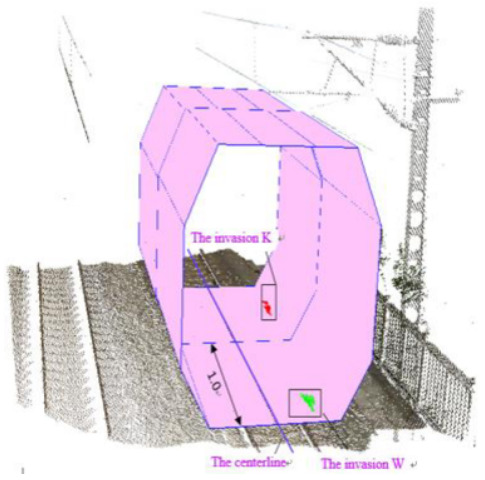

(b) The proposed algorithm

Figure 10. Part of the diagram of clearance inspection

Table 1. The statistics of the number of invasive distribution

\begin{tabular}{lccc}
\hline Dataset & Algorithm in Ref[9] & Algorithm in Ref[10] & Proposed algorithm \\
\hline Tunnel & 10 & 10 & 10 \\
Platform & 6 & 12 & 12 \\
Subgrade & 5 & 6 & 6 \\
\hline
\end{tabular}

The proposed algorithm and the method in Ref [10] find all invasive points, and 75\% invasive distribution are found by the method in Ref [9]. The invasion point $M$ that is not within the buffer could not be found as shown in Figure 10 (a).

The inspection time of the proposed algorithm is shorter than that of method in Ref [9] and the method in Ref [10] (Figure 11) and the efficiency is improved by $83.4 \%$. The Ref [9] spend a lot of time to structure TIN. In Ref [10], all point clouds are projected to the section in segments, which involves a large number of point clouds computing, resulting in low point cloud processing efficiency. In this paper, the proposed algorithm constructs the $\mathrm{AABB}$ of the clearance polyhedron to complete the preliminary clearance inspection, reduce a large number of point clouds computation.

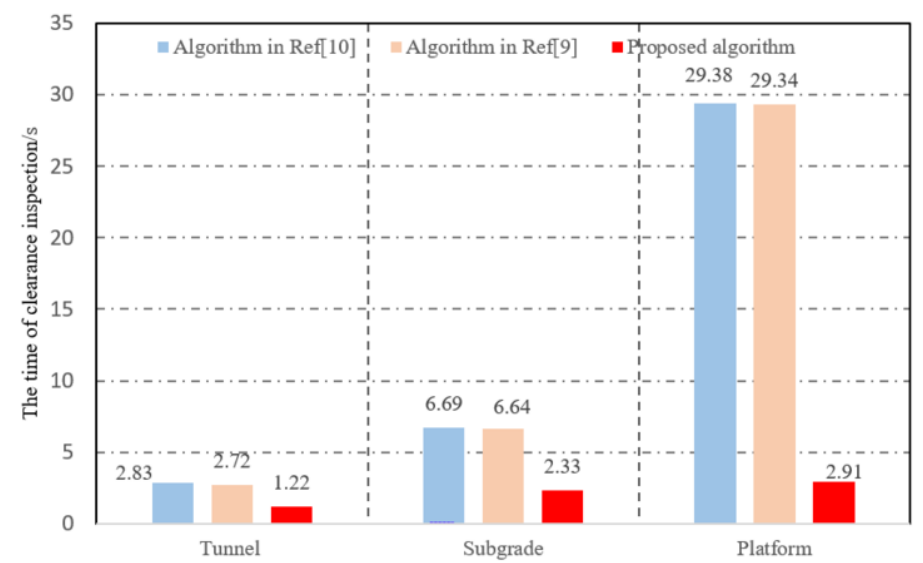

Figure 11.The time of the experiment 


\section{Conclusion}

The proposed algorithm based on the topology relationship between vehicle LiDAR point cloud and polyhedron, which can quickly complete the clearance inspection of all positions in the railway point cloud space and solve the problem of low accuracy and low time efficiency of railway clearance inspection based on LiDAR point cloud.

By comparing and analyzing the accuracy of experimental results of method in Ref [9] and the proposed algorithm, it can be seen that the proposed algorithm continuously completes the whole clearance inspection along the railway centerline direction with high accuracy .Through the analysis of the time of section clearance method and the proposed algorithm, this algorithm has obvious advantage in time efficiency. At the same time, In the process of the proposed algorithm, the user only needs to set the data such as the starting mileage of the railway, the railway centerline and the length parameters of the clearance polyhedron before detection, then the clearance inspection can be completed automatically, which greatly improves the efficiency. The experiment shows that the proposed algorithm has the advantages of high accuracy and high efficiency, which is suitable for railway clearance inspection based on LiDAR point cloud.

Due to the high accuracy and efficiency of the clearance inspection algorithm in this paper, it can be considered to combine with LiDAR point cloud data acquisition system to realize real-time dynamic clearance inspection of railway.

\section{References}

[1] Xiong X,Gao S, Chen H, Zhou Q, He Z. Principle and realization method of tunnel deformation detection based on image recognition and data transmission technology. Engineering Sciences, 2010, 8(4): 23-25

[2] Li P. Tunnel detection based on image processing. Beijing Jiaotong University, 2006.

[3] $\mathrm{Hu} \mathrm{Q}$, Chen $\mathrm{Z}, \mathrm{Wu} \mathrm{S}$. Fast and Automatic railway building structure clearance detection technique based on mobile binocular stereo vision. Journal of the China Railway Society, 2012, 34(1): 65-71.

[4] Chen M,He Y,Gong P,Li Z,Lu H. A close-range detector camera calibration method with high close angle. Journal of Railway Science and Engineering, 2019, 16(3): 796-803.

[5] Zhan D, Yu L, Xiao J, Chen T. The calibration approach study on the multiple vision sensors for railway tunnel clearance full cross-section inspection. Journal of the China Railway Society, 2015, 37(7): 98-106.

[6] Soilán M, Sánchez-Rodríguez A, del Río-Barral P, Perez-Collazo C, Arias P, Riveiro B. Review of laser scanning technologies and their applications for road and railway infrastructure monitoring. Infrastructures, 2019, 4(4).

[7] Puente I, Akinci B, González-Jorge H,Díaz-Vilariño L, Arias P. A semi-automated method for extracting vertical clearance and cross sections in tunnels using mobile LiDAR data. Tunneling and Underground Space Technology, 2016, 59:48-54.

[8] Gargoum S A, Karsten L, El-Basyouny K, Koch J C. Automated assessment of vertical clearance on highways scanned using mobile LiDAR technology. Automation in Construction, 2018, 95:260-74.

[9] Zhou Y, Wang S, Mei X,Yin W, Lin C, Hu Q, Mao Q. railway tunnel clearance inspection method based on 3d point cloud from mobile laser scanning. Sensors (Basel), 2017, 17(9): 1-20.

[10] Niina Y, Honma R, Honma Y, Kondo K, Tsuji K, Hiramatsu T, Oketani E. Automatic rail extraction and clearance check with a point cloud captured by MLS in a railway. ISPRS International Archives of the Photogrammetry, Remote Sensing and Spatial Information Sciences, 2018, XLII-2:767-771. 